MS18-P03

\section{Structural behaviour of Copper(I) Iodine compounds under high pressure}

Javier Gonzalez-Platas ${ }^{1}$, Amagoia Aguirrechu-Comeron ${ }^{1}$, Ulises R. Rodriguez-Mendoza ${ }^{1}$, Victor Lavin ${ }^{1}$, Sandrine Perruchas ${ }^{2}$

1. Departamento de Fisica. Universidad de La Laguna, La Laguna, Spain

2. Institut des Matériaux Jean Rouxel, Nantes, France

email: jplatas@ull.edu.es

Copper(I) iodine compounds can exhibit interesting mechanochromic and thermochromic luminecest properties with important technological applications. This fact is due two mainly factors. The first is about the large variety of structural configurations as staircase ladders linked by briding $\mathrm{N}$-donor ligands or cubane clusters with phospine ligand [12 ] among others. The second is the behaviour of these compounds under external stimuli inducing structural changes on bond distances, dihedral and/or torsional angles and packing between molecules. There are a lot of studies where this external stimuli is the temperature and how it affect the interactions of $\mathrm{Cu}$...Cu. Other similar studies are based on mechanical stimuli as grinding but only a few are using the pressure. We are reporting the structural and luminescence studies under high pressure, at room temperature under high pressure, of three copper iodine samples with different configuration. A cubane cluster type $\mathrm{Cu} 4 \mathrm{I} 4\{\mathrm{PPh} 2(\mathrm{CH} 2=\mathrm{CH} 2)$, $1 \mathrm{D}$ coordination polymer (staircase) $\mathrm{CuI}(6-$ methylquinoline) and 2D coordination polymer (staircase). For such compounds we present an analysis about their compressibility and bulk modulus through isothermal Equation of State calculations [3]

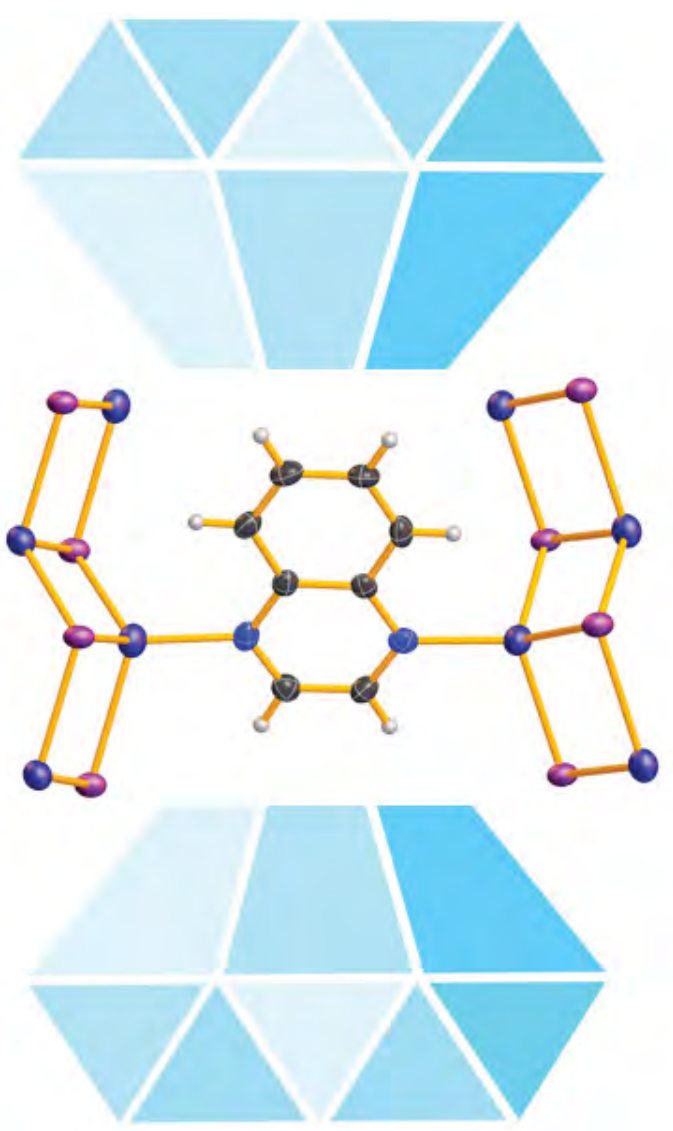

References:

[1] Q. Benito, I. Maurin, T. Cheisson, G. Nocton, A. Fargues, A. Garcia, C. Martineau, T. Gacoin, J.-P. Boilot, and S. Perruchas Chem. - A Eur. J. 2015, 21, 5892-5897.

[2] A. Aguirrechu-Comerón, R. Hernández-Molina, P. RodríguezHernández, A. Muñoz, U. R. Rodríguez-Mendoza, V. Lavín, R. J. Angel, and J. Gonzalez-Platas, Inorg. Chem. 2016, 55, 7476-7484. [3] J. Gonzalez-Platas, M. Alvaro, F. Nestola, and R. Angel, J. Appl. Crystallogr. 2016, 49, 1377-1382.

Keywords: Copper(I), Pressure, EoS 